\title{
The Energy Landscape of Negatively Charged BSA Adsorbed on a Negatively Charged Silica Surface
}

Karolina Tokarczyk ${ }^{1}$, Karina Kubiak-Ossowska ${ }^{*}$, Barbara Jachimska ${ }^{1}$, Paul A. Mulheran $^{2}$

${ }^{1}$ Jerzy Haber Institute of Catalysis and Surface Chemistry (PAS), Niezapominajek 8, 30-239 Cracow, Poland,

${ }^{2}$ Department of Chemical and Process Engineering, University of Strathclyde, James Weir Building, 75 Montrose Street, Glasgow G1 1XJ, UK. 


\begin{abstract}
We study the energy landscape of the negatively charged protein BSA adsorbed on a negatively charged silica surface at $\mathrm{pH} 7$. We use fully atomistic molecular dynamics (MD) and steered MD (SMD) to probe the energy of adsorption, and the pathway for the surface diffusion of the protein and its associated activation energy. We find an adsorption energy $\sim 1.2 \mathrm{eV}$, which implies that adsorption is irreversible even on an experimental timescales of hours. In contrast, the activation energy for surface diffusion is $\sim 0.4 \mathrm{eV}$, so that it is observable on the $\mathrm{MD}$ simulation timescale of $100 \mathrm{~ns}$. This analysis paves the way for a more detailed understanding of how a protein layer forms on biomaterial surfaces, even when the protein and surface share the same electrical polarity.
\end{abstract}




\section{INTRODUCTION}

Protein adsorption phenomena at solid surfaces have received much interest in industrial and biomedical processes. For example, in recent years, increasing attention has been focused on areas such as biochemical sensors, biofilm fouling, biocompatible materials, medical implants and drug delivery devices. ${ }^{1-3}$

While nonspecific protein adsorption on surfaces can cause serious problems such as degrading the analytical performance of devices, it greatly enhances our knowledge of the protein adsorption to interfacial regions. ${ }^{4-9}$ Protein adsorption is well known to be dependent on environmental factors, for example $\mathrm{pH}$, ionic strength, and also physicochemical properties of the protein and surface. ${ }^{4,7,10}$ Studies concerning protein adsorption onto charged substrates show that the major driving forces are electrostatic and hydrophobic interactions; $;^{3,5,11-13}$ these govern the specific orientation and the structure of the proteins in the adsorbed layers. As well as quantifying molecular orientation, conformation or aggregation of adsorbed protein, it is also important to quantify dynamic phenomena such as surface diffusion, which can affect the surface excess density. ${ }^{8,14,15}$ Since the surface processes can change the protein biochemical activity, many questions still need to be addressed regarding protein interfacial behavior.

Serum albumins are one of the most abundant proteins in blood, therefore interfacial behavior studies of proteins such as human (HSA) and bovine (BSA) serum albumins seem to be crucial for biomedical applications. ${ }^{1-3}$ Due to its high similarity to HSA, low cost and availability, BSA is often used as a model protein. It consists of 583 amino acids; the molecular mass is $\sim 67 \mathrm{kDa}$. The protein's charge distribution is inhomogeneous which makes adsorption on both positively and negatively charged surfaces possible. Silica surfaces are a common reference for studying protein - hydrophilic/charged/inorganic surface interactions. Silica is a biocompatible and biodegradable material, which can be used in many pharmaceutical 
applications i.e. as a possible drug delivery device for a therapeutic protein or immobilized biocatalysts. ${ }^{13,16-18}$ In a wide range of $\mathrm{pH}$ it is negatively charged with deprotonated silanol groups ${ }^{4}$ and one of its important features is long-term stability. When a solid surface is in contact with a protein solution, at steady state the material is covered with adsorbed protein molecules. Hence it is important to understand how the proteins interact with the hard material surface.

Insight into protein adsorption processes involved in biotechnological applications is essential to achieve materials with high biocompatibility and good performance. ${ }^{1,8}$ However, protein - inorganic solid surface interactions are sometimes difficult to analyze from experiments and need to be additionally revealed through computational techniques. ${ }^{19-22}$ Simulations have turned into an essential tool to provide insights from the atomistic level to validate and interpret experimental work. Such an approach allows us to elucidate structural and dynamical details with deep understanding of the molecular mechanism of adsorption, including the energy of adsorption, diffusion on the surface and desorption.

A detailed description of the adsorption dynamics can be provided by computational methods such as Molecular Dynamics (MD) and its variants such as Steered Molecular Dynamics (SMD). ${ }^{5,19-21,23-25}$ Vilhena et al. ${ }^{19}$ used atomistic MD and SMD to study the difference between free and forced adsorption of BSA onto graphene in terms of protein secondary structure, contact area and protein spreading. Their results show that free adsorption occurs with only minimal structural changes of BSA. Even if adsorption was forced, the BSA was able to preserve the structural properties of the majority of its binding sites. In another study, Mücksch et al. ${ }^{23}$ studied adsorption and forced desorption of BSA and lysozyme with a model, highly hydrophobic graphite surface using MD and SMD simulations. They found that BSA loses its secondary structure during adsorption and also becomes almost fully unzipped during pull-off, presumably due to the hydrophobicity of the surface. Wei et al. ${ }^{25}$ demonstrated 
the need for long-time atomistic simulation in order to gain a complete understanding of the protein adsorption process and also showed that constant improvement in simulation methodologies enables this endeavor. Therefore, the purpose of our work is a detailed protein interfacial behavior analysis, including structural characteristics and dynamics.

In this paper we report the interactions between a model, negatively charged silica surface and a negatively-charged bovine serum albumin (BSA) protein at $\mathrm{pH}$. As discussed previously, electrostatic repulsion makes this a challenging system for modelling, ${ }^{5,11}$ despite the experimental evidence that the protein readily adsorbs to the silica surface. ${ }^{4,11}$ We have found that the inhomogeneous nature of the charge distribution across the protein surface enables the adsorption process, in conjunction with the electrostatic screening provided by the diffuse layer of counter ions at the charged silica surface. Here we study the adsorptiondesorption of the BSA with MD and SMD techniques, in order to analyse the energy required for BSA adsorption and desorption processes. We also investigate the surface diffusion pathways for the adsorbed protein and the associated energy landscape, adding to our understanding of the protein dynamics at the water-silica interface.

\section{MATERIALS AND METHODS}

\section{Simulations Studies}

All our simulations were carried out using the NAMD 2.8 ${ }^{26}$ simulation package together with the CHARMM27 force field and analyzed using $\mathrm{VMD}^{27} \cdot 3 \mathrm{~V} 03^{28}$ was used as the starting BSA structure. As in our previous simulations, ${ }^{5,11}$ the protein $(-17 \mathrm{e})$ and surface slab $(-429 \mathrm{e})$ charge were neutralized by $\mathrm{NaCl}$ at an ionic strength $\mathrm{I}=5 \times 10^{-2} \mathrm{M}$. This step is necessary due to the Particle Mesh Ewald (PME) method, which is the infinite sum of charge-charge interactions 
and converges well only in the case of neutral systems. ${ }^{26}$ Moreover, the ions provide screening of the electrical field created both by the surface and protein. As discussed elsewhere, ${ }^{5,11}$ without local electric field fluctuations and screening of both the $\mathrm{BSA}$ and $\mathrm{SiO}_{2}$, the $\mathrm{BSA}$ adsorption on the $\mathrm{SiO}_{2}$ surface would not be feasible in a reasonable simulation time scale.

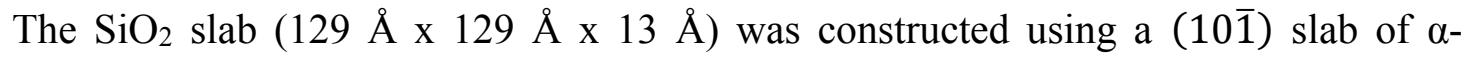
crystabolite cut from a bulk crystal in such a way to leave siloxide groups $\left(\equiv \mathrm{SiO}^{-}\right)$both at the top and bottom of the slab, so that the slab has a net negative charge but zero dipole moment. The resulting surface charge density, with partial charges on the $\mathrm{O}(-0.55 \mathrm{e})$ and $\mathrm{Si}(1.1 \mathrm{e})$ atoms, mimics the negative zeta potential at $\mathrm{pH} 7$ observed experimentally. ${ }^{4}$ It is worth noting that, in the MD methodology, the $\mathrm{pH}$ is set a priori at a particular level through the selection of the charge states of the silica surface and protein residues. The force field parameters (including protonation states of particular side chains and water molecules) are typically designed to reproduce the physiologically important $\mathrm{pH} 7$. During the simulation, due to the force field used, bonds cannot be created or broken, and therefore $\mathrm{H}^{+}$and $\mathrm{OH}^{-}$moieties are not present in the simulation. Hence, the silica-water chemistry is not reproduced precisely, but nevertheless the water behavior and creation of water layers ${ }^{4,5}$ mimics well the detailed chemistry; the method ignores fast chemistry while giving a good insight into slower processes such as protein adsorption on a given surface.

The neutral system is used in a simulation cell of size $129 \AA$ x $129 \AA$ x $191 \AA$, filled by water (we use the TIP3P model) with periodic boundary conditions. In its starting orientation, the BSA's intrinsic dipole moment points towards the negatively charged surface (with an angle of about $45^{0}$ to the normal) favoring adsorption to the surface. More details regarding the electrostatics of the designed system can be found in our previous reports. ${ }^{5,11}$ 
The simulation cell was subjected to minimization, heating and $200-500$ ns production trajectories at a constant $300 \mathrm{~K}$ temperature maintained by a Langevin thermostat. Additionally, we have used the PME method to calculate the electrostatic interactions, while the cut-off for van der Waals interactions was $12 \AA$. To reduce computational costs, water molecules were treated as a rigid bodies, and a timestep of 2 fs was used. We have identified a successful adsorption trajectory previously ${ }^{5,11}$.

In this work, we have extended our analysis for two additional non-adsorption trajectories to obtain an estimate of the adsorption energy. All three trajectories are independent runs from the same starting configuration; since the evolution of the system is stochastic in nature, these are not identical trajectories. The BSA trajectories are denoted as: AD, which is the $500 \mathrm{~ns}$ long simulation where BSA adsorbed on our model silica surface, ${ }^{.511} \mathrm{~N}-\mathrm{AD} 1$ and NAD2 which are $200 \mathrm{~ns}$ trajectories where adsorption has not been observed.

\section{SMD Simulations Studies}

SMD simulations started from the existing AD adsorption trajectory at $375 \mathrm{~ns}$. We used this adsorption state in order to start our series of SMD simulations which investigate the impact of pulling on desorption and diffusion processes. AD-375ns is a structure showing stable BSA adsorption at the silica surface, as described in our previous work and denoted as state F (final adsorption state). ${ }^{5,11}$ For clarity, we use the same description in this work. Thus, the BSA adsorption stage notations used in this work are: (i) Stage F - BSA is adsorbed and Lys537 side chain penetrates the inner water layer; (ii) Stage F' - BSA is adsorbed and Lys537 side chain penetrates the outer water layer; (iii) Stage M - BSA is mobile (diffuses) on the surface, but doesn't desorb, and the Lys537 side chain lies just above the outer water layer; (iv) Stage D BSA is desorbed. The state F has been obtained for one BSA molecule adsorbed to the silica 
surface. $^{5,11}$ The molecule was oriented with its IIIB subdomain towards the silica surface and the Lys537 side chain penetrating both water layers and creating a strong anchor to the surface. The list of key residues for the BSA-silica surface interactions (state F) includes: Glu494, Thr495, Lys535, Lys537, Thr539, Glu541, Gln542, Thr580, and Ala583. ${ }^{11}$

In the SMD simulations performed in this study, while the MD parameters remained unchanged, we have applied an external force with constant velocity pulling of $0.005 \AA / \mathrm{ps}$ and with a spring constant $278 \mathrm{pN} / \AA$. In practice, the SMD protocol means that we introduce one or more dummy atoms which are attached to the chosen protein atoms by virtual springs, then we pull with constant velocity and measure the force between the dummy and protein atoms. ${ }^{26}$ We use 10 ns duration simulations to probe desorption and diffusion effects. In total, we have performed 12 SMD runs, they differ in the pulled residue side chain atom as well as the pulling direction. An external force was applied in four directions: away from the surface in the $-\mathrm{x}$ direction which is denoted in this work as " $u$ " (up); across the surface, which is denoted as "a" (across): in -y direction ("a1"), +z direction ("aa") and -y;+z direction ("a 3 ").

Knowing the list of key residues for the BSA-silica surface interactions ${ }^{5}$, we decided to use these residues in the SMD runs, namely the pulled atoms (in various runs) were the following: Glu494 C $\alpha$, Thr495 C $\alpha$, Lys535 C $\gamma$, Lys537 C $\gamma$, Thr539 C $\alpha$, Glu541 C $\gamma$, Gln542 C $\gamma$, Thr580 C $\alpha$, and Ala583 N.

First, we have performed 4 SMD runs, where we pull only the Lys537 side chain (using C $\gamma$ ) up (trajectory Lys537_u) and across the surface (trajectories: Lys537_a 1 , Lys537_a Lys537_a3). Then, we have run another 4 SMD simulations in the same directions as above, and pull all of the key residues apart from Lys537 (trajectories: all_noLys537_u, all_noLys537_a 1 , all_noLys537_a 2 , all_noLys537_a3). Finally, we have used an external force to pull all of the key residues together and denote these trajectories as: all_u, all_a $\mathrm{a}_{1}$, all_ $\mathrm{a}_{2}$, 
all_a a $_{3}$. We estimated the energy of diffusion and adsorption from the appropriate force-distance curves.

To get information about protein re-adsorption we have also performed four additional 10 ns duration MD simulations following these SMD trajectories: Lys537_u, Lys537_a Lys537_a2, and Lys537_a3. The runs were starting from a priori chosen time moments of the SMD simulations to check the protein behavior when the external force is released. The time chosen is when the BSA molecule is close to the surface, however the Lys537 side chain is in various desorbed stages: 2.24 ns for Lys537_u, 3.18 ns for Lys537_a, 2.10 ns for Lys537_a and 2.52 ns for Lys537_a 3 .

\section{Energy Analyses}

We perform a series of atomistic MD simulations in order to calculate the adsorption energy of the protein to the silica surface. The adsorption energy is estimated by comparing the total energy of the system with the protein in bulk water above the surface, with that of the system once the protein has adsorbed. We take into account the relaxation of the protein structure in the solvent as explained below. This approach provides us with the desired energy estimate, neglecting changes to entropic contributions. ${ }^{29}$

We also perform the series of SMD studies described above to estimate the activation energy for diffusion across the surface ${ }^{24}$. We note that because the adsorbed protein is anchored to the surface by key residues' side-chains, the protein diffusion across the surface necessitates the partial desorption of these side chains. This is very similar to the situation previously investigated with lysozyme adsorbed to a model charged surface ${ }^{24}$. By performing the SMD trajectories slowly with modest spring constant, we explore the possible pathways and energy 
landscape for the side-chain desorption, and hence find the mechanism for free (un-forced) surface diffusion for the adsorbed BSA.

\section{RESULTS AND DISCUSSION}

\section{BSA adsorption energy analysis}

As described in the Methods section, we analyze three trajectories, one for successful adsorption (AD) and two for no adsorption (N-AD1 and N-AD2). The differences between trajectories reflect various local minima that the protein reached during the preparation period, as expected in MD simulation; the same starting geometry, due to thermal randomization, will not lead to exactly the same results. Apparently, in the case of BSA and the model $\mathrm{SiO}_{2}$ surface, there are various local minima which do not lead to the protein-surface orientation, separation and electric field fluctuations that yield protein adsorption within a $100 \mathrm{~ns}$ timescale. However, we emphasize that all the structures obtained are energetically stable. The $33 \%$ success rate in the adsorption trajectories illustrates the fact that adsorption of a negatively charged protein onto a negatively charged surface is a rare process in the studied timescale, as already discussed..$^{5,11}$

Overlaps of the final protein configuration from each trajectory indicate that in all cases the overall BSA structure is stable and similar to each other (Fig. 1). Neither domain reorganization nor secondary structure change (such as $\alpha$-helix unfolding) are observed. When compared to the initial BSA structure, the smallest changes are noticed for the AD trajectory (Fig. S1). As shown in the Supplementary Materials (§S1), the structure of subdomain IIIB, where the adsorption site is located (see Fig. 1 inset), is very well maintained after the AD trajectory, while both N-AD trajectories show slightly higher structural flexibility in this region (Fig. 1). 
It might suggest that the surface stabilizes the structure of the IIIB domain, which is the most hydrophilic subdomain of BSA and one of the least negatively charged as discussed elsewhere. ${ }^{5,11}$ Structural similarity (further supported by RMSD and RMSF analyses provided in Supplementary Materials, §S1 and Fig. S2) indicates that within all trajectories studied we observe the naturally-occurring structural flexibility of BSA. Therefore, an energy comparison between $\mathrm{AD}$ and $\mathrm{N}-\mathrm{AD}$ trajectories might lead to quite accurate estimation of BSA adsorption energy on the model silica surface.

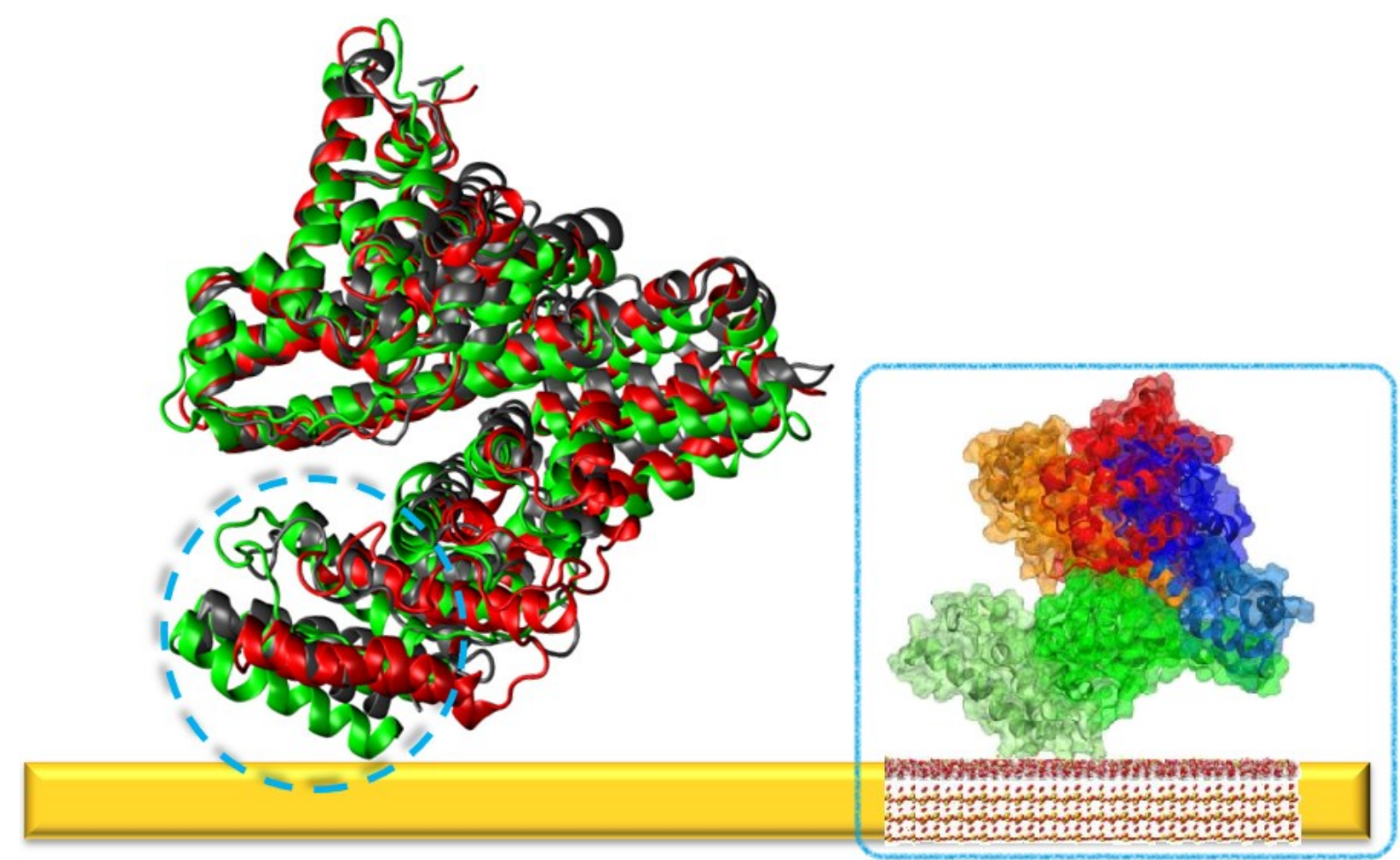

Figure 1. The main figure shows a BSA structure overlap after AD (black), N-AD1 (red) and N-AD2 (green) trajectories. For clarity the protein structure is shown by cartoon and the surface is represented by solid yellow rectangle. The dashed blue circle indicates the adsorption site (subdomain IIIB) while the blue squared inset shows adsorbed BSA structure on silica surface after $375 \mathrm{~ns}$ in the AD trajectory. In the inset, the BSA secondary structure is indicated as a cartoon, the protein surface is shown as a ghost surface colored by subdomain as introduced by Majorek et al. ${ }^{28}$ : IA, red; IB-orange; IIA-blue; IIB-light blue; IIIA-green; IIIB-lime. $\mathrm{SiO}_{2}$ 
surface atoms are shown by CPK representation (oxygen - red; silicon - yellow). The water layer is shown by transparent CPK. The bulk water and ions are not shown for clarity. For the cartoon, ghost and CPK representations, the VMD software definitions ${ }^{28}$ are used.

Plots of the total energy obtained within AD, N-AD1 and N-AD2 trajectories versus time (Fig. S3) show two stages: energy $\mathrm{E}_{1}$ is observed during the first 50 ns where the initial protein relaxation in the water occurs, and energy $E_{2}$ for the rest of the simulation time. Of course, the total energy includes the energy of surface-water, surface-ions, surface-protein, protein-water, protein-ion and water-ion interactions, as well as the internal energy of each subsystem. Nevertheless, due to the length of the trajectories and the identical numbers of species in each trajectory, we can assume that averages of those energies are the same within each system.

It is worth noting that even in the $\mathrm{AD}$ trajectory, the BSA spent the first $50 \mathrm{~ns}$ freely diffusing in the water, unaffected by the presence of the surface. For each trajectory we calculated the average values of $\mathrm{E}_{1}$ and $\mathrm{E}_{2}$ (along with standard errors) as well as the difference $\Delta \mathrm{E}$ in these (see Tab. 1). To avoid over-sampling of correlated data, we sample the energy every $0.2 \mathrm{~ns}$ to calculate the statistics. The energy difference $\Delta \mathrm{E}_{\mathrm{AD}}$ between the initial free diffusion (e.g. structure relaxation) and the established adsorption state in the AD trajectory is $2.1 \pm 0.2$ $\mathrm{eV}$ (Fig. 2), while $\Delta \mathrm{E}$ calculated for $\mathrm{N}-\mathrm{AD}$ trajectories (between initial relaxation and established free diffusion in the water) is $0.8 \pm 0.2 \mathrm{eV}$ and $0.9 \pm 0.2 \mathrm{eV}$ for N-AD1 and N-AD2 respectively (see $\S \mathrm{S} 2$ in the Supplementary Materials). $\Delta \mathrm{E}_{\mathrm{AD}}$ corresponds to the adsorption energy convoluted with the energy of long-term relaxation and normal free dynamics, while $\Delta \mathrm{E}_{\mathrm{N}-\mathrm{AD} 1}$ and $\Delta \mathrm{E}_{\mathrm{N}-\mathrm{AD} 2}$ corresponds to the energy of the long-term relaxation and normal free dynamics only. Therefore, the energy of protein adsorption, $\mathrm{E}_{\mathrm{A}}$ can be estimated from 


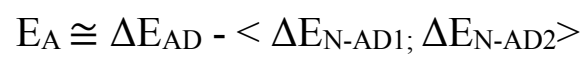

where $<\ldots>$ is an average value. Thus, $\mathrm{E}_{\mathrm{A}} \cong 1.25 \pm 0.4 \mathrm{eV}$ is our best estimate of the adsorption energy of BSA on the model $\mathrm{SiO}_{2}$ surface, notwithstanding the approximate nature of this calculation.

It is useful to consider this value of the BSA adsorption energy in terms of Arrhenius rates. ${ }^{24}$ For an activation energy of $1.25 \mathrm{eV}$, the Arrhenius rate is measured in years. Hence our estimated adsorption energy helps explain why in our simulations we have never observed spontaneous desorption.

There are 13 hydrogen bonds between key BSA residues and the water layers ${ }^{11}$ in adsorption state F; ten of them to the outer water layer (OWL) and three to the inner water layer (IWL). From that one can assume that the energy associated with hydrogen bonds would be equal $\sim 2.6 \mathrm{eV}\left(\sim 0.2 \mathrm{eV}\right.$ for each $\mathrm{H}$-bond), which is much higher the $\mathrm{E}_{\mathrm{A}}$ calculated above. However, in solution the BSA also has hydrogen bonds to the solvent, and the adsorption energy is the difference in energies between fully solvated protein in bulk solution and at the surface. Furthermore, other interactions (electrostatics and van-der-Waals) play a role in the energetics. Therefore, our estimate of $\mathrm{E}_{\mathrm{A}}$ appears reasonable.

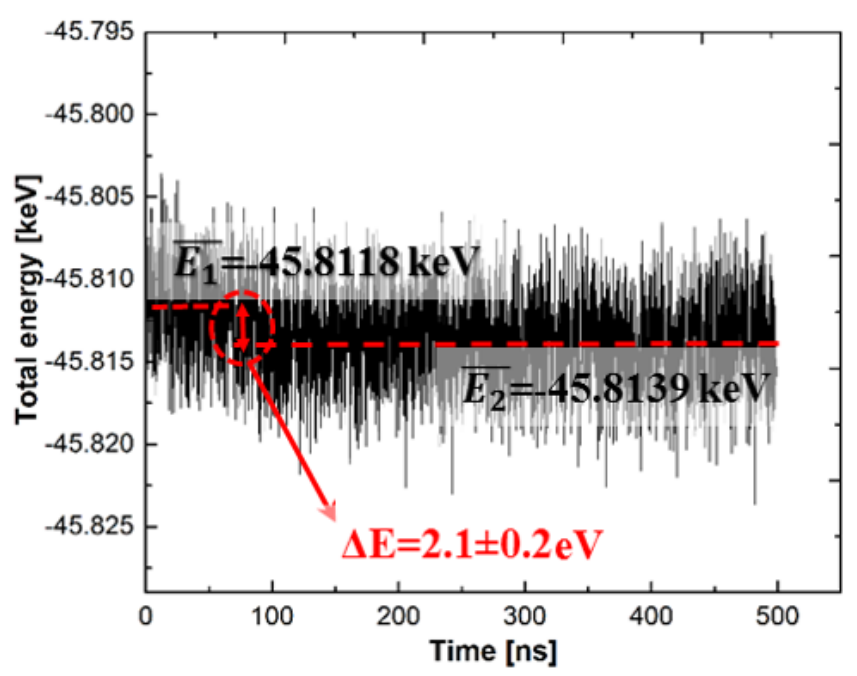


Figure 2. Total energy within the AD trajectory against time displayed as a single black trace (the gray shading is used to enhance the clarity of the annotation). The average of the two stages, $\mathrm{E}_{1}$ and $\mathrm{E}_{2}$, are labeled.

Table 1. The average total energies (with standard error estimations) obtained within the first $50 \mathrm{~ns}\left(\mathrm{E}_{1}\right)$ and the remaining time $\left(\mathrm{E}_{2}\right)$ of the $\mathrm{AD}$ and N-AD trajectories. The difference $\Delta \mathrm{E}$ is given in the last column.
$\mathbf{E}_{1}[\mathrm{keV}]$
$\mathrm{E}_{2}[\mathrm{keV}]$
$\Delta E[e V]$

\begin{tabular}{cccc}
\hline Adsorbed & $-45.8118 \pm 1.5 \cdot 10^{-4}$ & $-45.8139 \pm 5.5 \cdot 10^{-4}$ & $2.1 \pm 0.2$ \\
Non-adsorbed_1 & $-45.8015 \pm 1.5 \cdot 10^{-4}$ & $-45.8023 \pm 0.9 \cdot 10^{-4}$ & $0.8 \pm 0.2$ \\
Non-adsorbed_2 & $-45.7941 \pm 1.5 \cdot 10^{-4}$ & $-45.795 \pm 0.9 \cdot 10^{-4}$ & $0.9 \pm 0.2$
\end{tabular}

\section{SMD Simulations of the Desorption Pathway}

The desorption pathway for the BSA on the model silica surface is probed using SMD simulation. The chosen pulling velocity of $0.005 \AA / \mathrm{ps}$ allowed us to probe the desorption mechanisms on a nanosecond timescale without visibly affecting the protein structure (i.e. no protein unfolding). Our previous $0.5 \mu \mathrm{s}$ M adsorption trajectory (AD) ${ }^{11}$ provided a detailed description how the negatively charged $\mathrm{BSA}$ at $\mathrm{pH} 7$ adsorbs to the negatively charged $\mathrm{SiO}_{2}$ surface, and revealed a unique orientation with preserved secondary and tertiary structure. Here, we use SMD results to add more details to the protein behavior in adsorbed stages F, F' and M 
as described above. In Fig. 3 we show the protein structure of the BSA adsorption trajectory (AD), when the BSA is in its final adsorption stage F. We observed that the IIIB subdomain, which is slightly negative overall in comparison to other subdomains and relatively hydrophobic, is attracted to the silica surface. Figure 3 also shows the inhomogeneous distribution of charged residues across the protein surface, and positive residues are seen to facilitate the adsorption to the negatively charged silica surface. The figure also displays the dipole moment of the protein, showing how it aligns in the electric field above the surface. The adsorption is strong and irreversible., 5,11

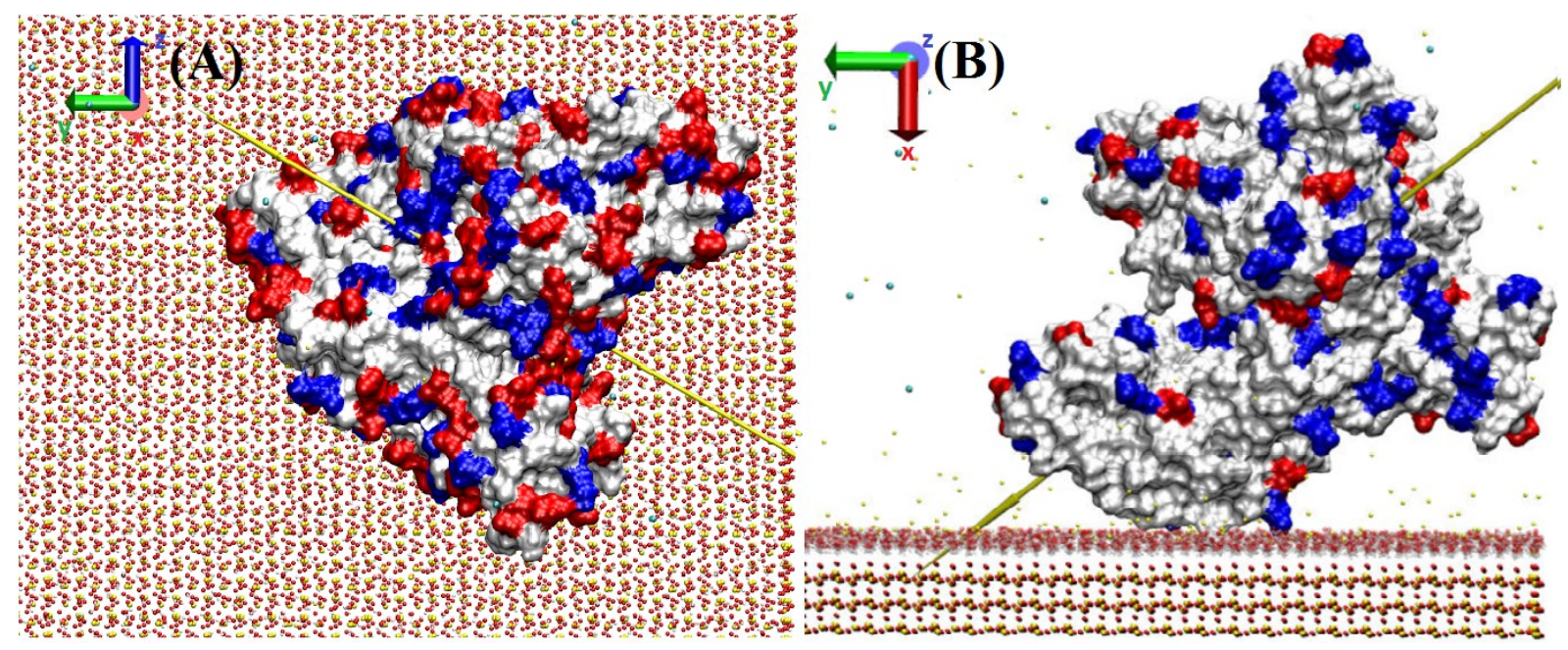

Figure 3. Molecular model of BSA on silica surface (A) top view (B) side view after $375 \mathrm{~ns}$ AD trajectory (representative for stage F). The protein surface is colored by residues' total charge (positive - blue, negative - red, neutral - white). The silica surface and surface-water are shown by transparent CPK (the bulk water is not shown for clarity). CPK color code: oxygen-red; silicon-yellow; hydrogen-white; chlorine-cyan; sodium-yellow. The yellow arrow shows the protein's dipole moment. 
During protein adsorption we usually observe an anchoring residue penetrating through the surface water layers; these are defined as well-ordered layers (of thickness $\sim 1.5 \AA$ ) located in close proximity to the silica surface, with the waters exposing hydrogen towards the silica oxygens. ${ }^{11}$ Our previous simulations showed that only the positively charged Lys537 residue plays this anchoring role effectively and penetrates through both surface water layers (see Figure 4 state $\mathrm{F})^{5}$ and so we pull the $\mathrm{C} \gamma$ atom of Lys 537 . We investigate the diffusion pathways of BSA by pulling in directions normal to the surface (trajectory Lys537_u) and parallel to the surface (trajectories Lys537_a1,2,3).

The desorption mechanism observed is similar for all trajectories, and here we discuss one exemplar, Lys537_u. Pulling the BSA up from the surface provides data directly corresponding to the adsorption energy of the main anchoring residue, while the energy barriers calculated from trajectories "a" (across the surface), additionally include the energy required for water layer reorganization. However, the energies are convoluted and it is not clear which part of the energy refers to which particular process.

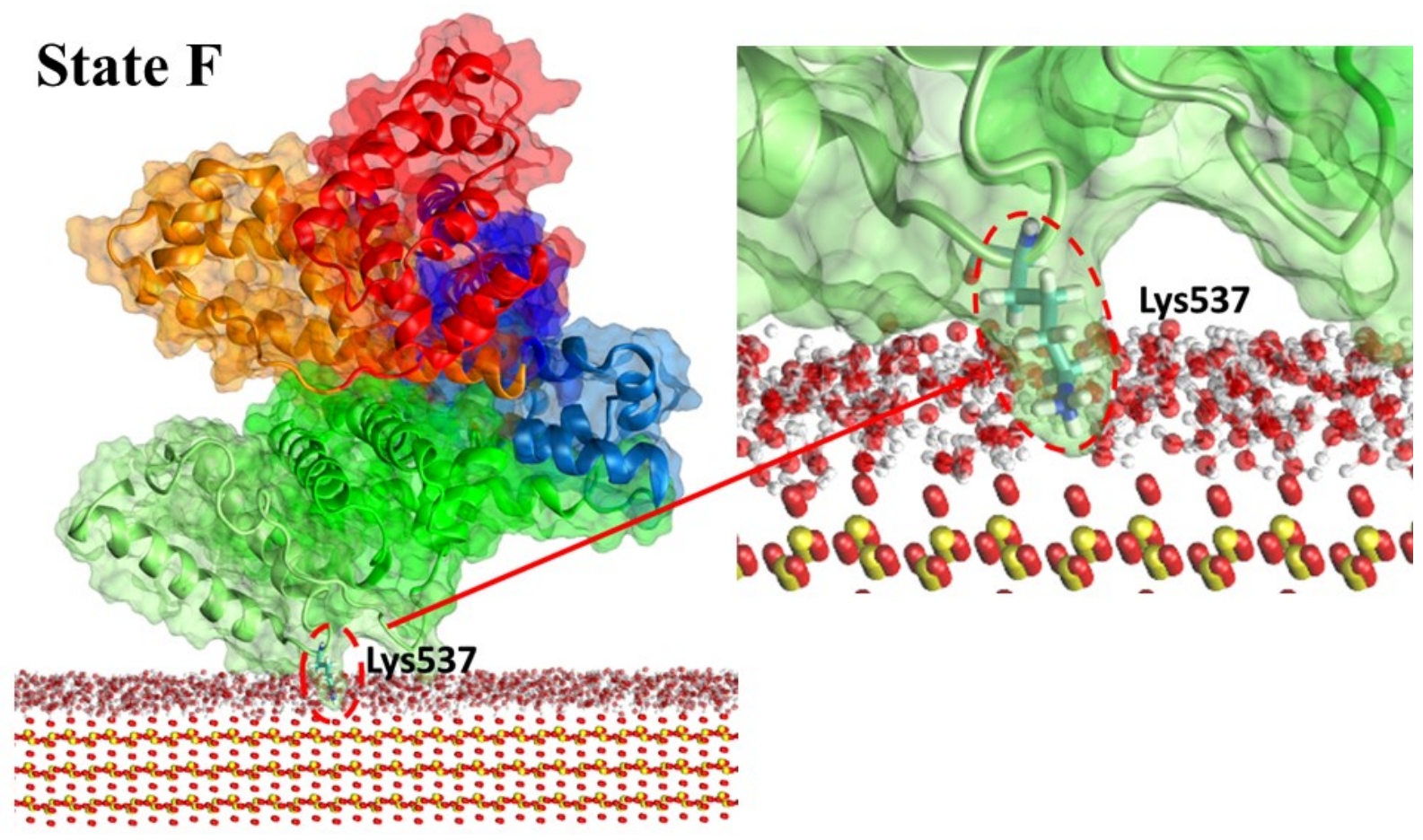


Figure 4. The surface adsorbed state of BSA at 375ns of the AD trajectory in which the Lys537 side chain penetrates the inner water layer (state F). Representation and color code follows the one introduced in Fig. 1. The Lys537 residue is annotated and indicated by licorice colored by name (hydrogen-white; carbon-cyan; nitrogen-blue).

From the trajectory Lys537_u, we can describe the Lys537 desorption process from the model silica surface in three steps (see Fig. 5). First, the Lys537 side chain from the initial state F moves out of the inner water layer at $0.22 \mathrm{~ns}$ to what we denote as state $\mathrm{F}$ ' in Fig. 5A. Then, at $0.58 \mathrm{~ns}$, it lies just above the outer water layer and interacts only with this layer, which corresponds to state $\mathrm{M}$ in our previous work ${ }^{11}$ (Fig. 5B). Our previous MD simulations showed two hydrogen bonds between the key BSA residue and the inner water layer at stage $\mathrm{F}^{5}$ These two H-bonds between Lys537 and the water layers are also present during the transition from state $\mathrm{F}$ to F', and from state F' to M. At 1.10 ns Lys537 completely loses contact with the outer water layer and no further residues anchor the protein to the surface. After this time, the BSA molecule moves away from the silica surface following the external force, and we denote this state as D (Fig. 5C). The protein-surface separation is now $\sim 10 \AA$.

The protein is now desorbed, but it is in the orientation which is close to the preferred one for adsorption, and when the external force is released the protein adsorbs again in the way already observed previously ${ }^{11}$ and achieves the adsorbed state $\mathrm{F}$ just as in the original MD trajectory. Due to the strong attraction to the silica surface, the BSA adsorbs again within the MD simulation time of $10 \mathrm{~ns}$. First, the Lys537 side chain penetrates the outer water layer $(\sim 0.2$ ns after the force release). After $\sim 5.0 \mathrm{~ns}$, it strongly interacts with the inner layer and then directly with the $\mathrm{SiO}_{2}$ surface as in state $\mathrm{F}$ of Fig. 4. The BSA re-adsorption process is observed in all our unbiased MD simulations, provided the initial distance of the protein's closest residue 
to the silica support was $\leq 8 \AA$, regardless of whether the Lys537 side chain was pulled along the normal or parallel to the surface. The BSA attraction to the silica is dominant and, in each trajectory with the external force released, the protein started its adsorption process immediately. The protein orientation does not change and it always comes back to the state before the start of the SMD simulated pulling (namely state F). This implies that either the state $\mathrm{F}$ orientation is the only one possible for adsorption, or that there was not enough time and space for the BSA to find another one within the conditions of strong attraction to the surface. From the original AD trajectory, when the protein adsorbed, desorbed and re-adsorbed again in stage $\mathrm{F}^{5,11}$ we are led to believe that the former option is more likely than the latter.

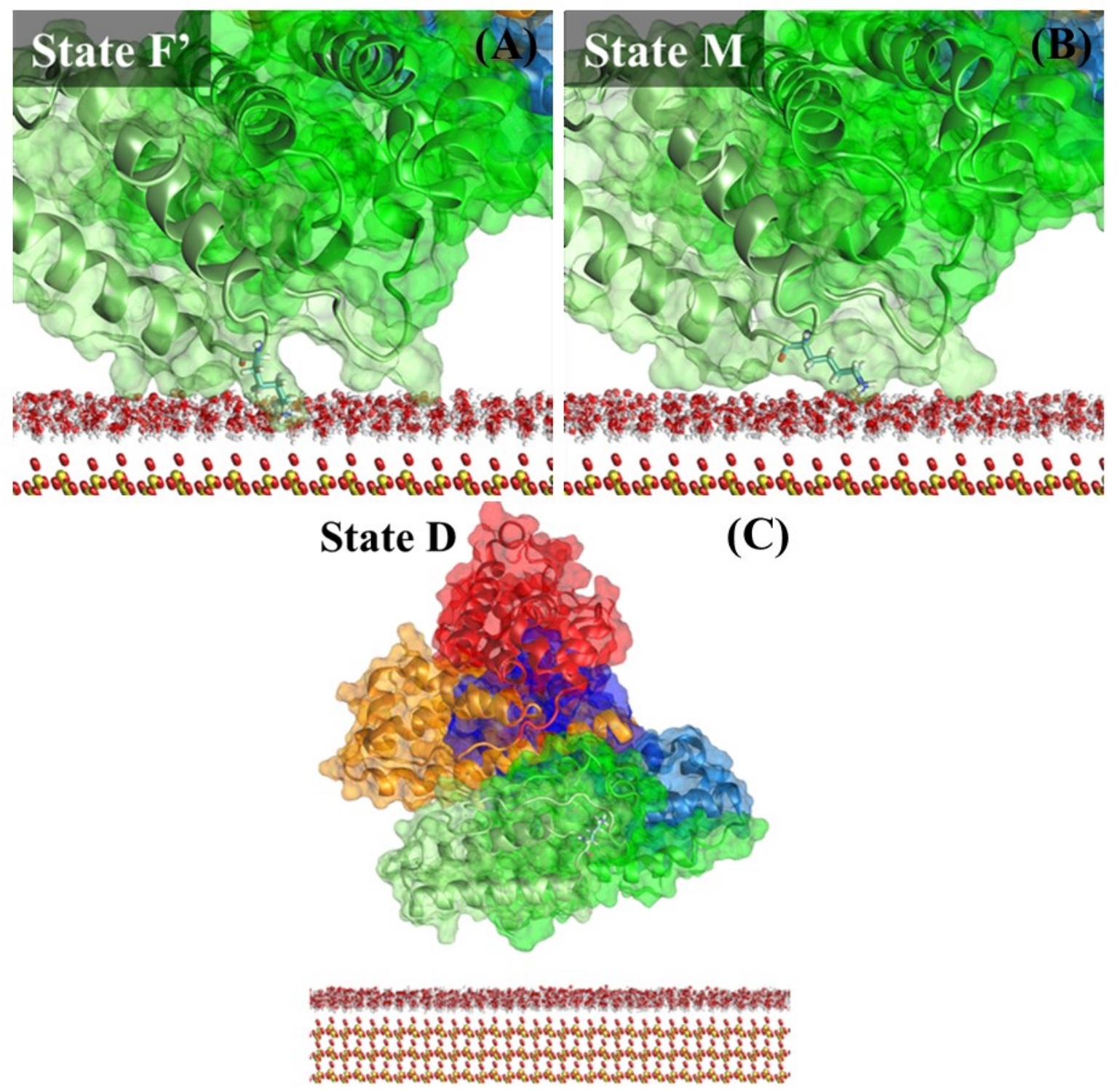


Figure 5. The states of BSA interacting with the silica surface: (A) State F' - Lys537 moves out of the inner water layer; (B) State M - Lys 537 lies just above the second water layer; (C) state D - the BSA is desorbed from the surface.

It is interesting that both in our previous ${ }^{5,11}$ and current work we can observe state $\mathrm{M}$, where BSA diffuses on the surface. The protein lateral diffusion on solid surfaces represents one of many dynamic phenomena important to protein layer formation; note that mobility does not necessarily require the entire molecule to desorb. ${ }^{30}$ There is no evidence of spontaneous protein desorption both in experimental $4^{4,11}$ and theoretical ${ }^{5,11}$ studies, suggesting that the migration of BSA molecule is due to surface diffusion rather than desorption. Moreover, the adsorption energy calculated above $(1.25 \pm 0.4 \mathrm{eV})$ suggests that spontaneous desorption should not be expected.

If the adsorbed BSA is indeed mobile on a surface it can profoundly affect the surface excess concentration. It might violate the random sequential adsorption (RSA) model tenets, which describe irreversible adsorption of immobile, non-interacting and nonoverlapping particles. ${ }^{31}$ Laterally mobile protein can rearrange itself after the initial adsorption process and thereby gain more efficient, densely packing arrangements and consequently higher surface coverage.

The phenomena described above was observed under laboratory conditions by MP-SPR experiments ${ }^{11}$ indicating the protein surface coverage grew to a maximum of $82 \%$ of a complete RSA monolayer. One can assume that such a big value for a negatively charged molecule adsorbed to a negatively charged surface is supported by protein lateral diffusion on the silica surface. As total internal reflection fluorescence (TIRF) and fluorescence recovery techniques indicate, the mobility of biomolecules at interfaces is observed to be an important process. ${ }^{14}$ 
To verify the conclusions which we draw from our majority choice of pulling the single $(\mathrm{C} \gamma)$ atom of Lys537, we have studied further trajectories named "all" and "all_noLys537", where we pulled atoms from key residues for the BSA-silica surface interactions. Of the list of key residues (see Methods section), Glu is negatively charged, Lys is positively charged, Gln and Thr are neutral, and Ala possesses a negative partial charge. All these residues, apart from the Ala side chain, are hydrophilic and able to interact with water. Only Lys537 penetrates through both surface water layers, whereas the other key residues are just above the outer water layer (see Fig. 6).

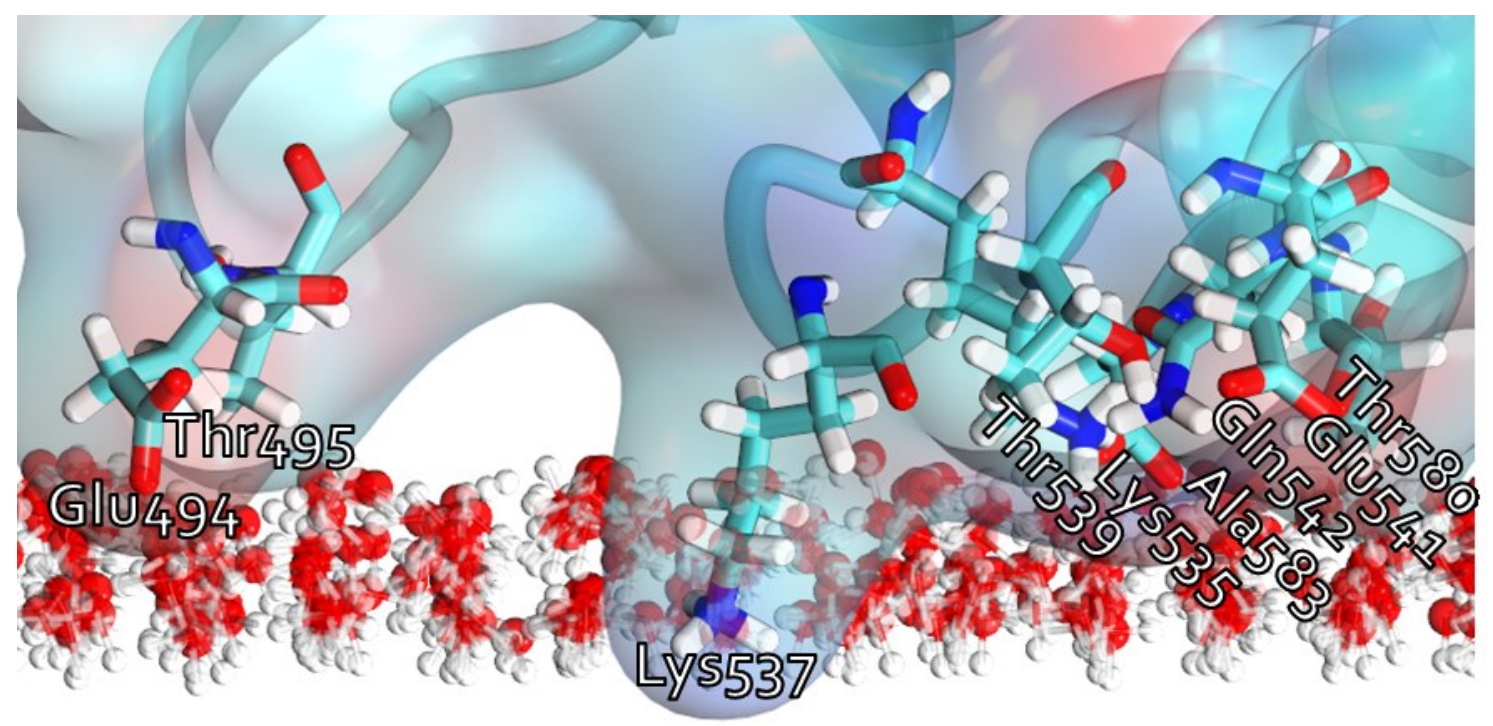

Figure 6. The surface adsorbed state of BSA at the end of an adsorption simulation (375 ns), in which all the key residues: Glu494, Thr495, Lys535, Lys537, Thr539, Glu541, Gln542, Thr580, and Ala583 are denoted.

First, we pull all residues that act as surface anchors for the BSA without Lys537 away from the surface (trajectories all_noLys537_u and all_noLys537_a). The trajectories all_noLys537_a did not reveal any substantially new features for the BSA desorption mechanism. Thus, we focus on the one case, in which residues are pulled in the normal direction 
in trajectory all_noLys537_u. The first residues which desorbed are Thr495 and Glu494. Then Thr539 and Glu541 desorbed from the surface and finally Lys535, Gln542, Thr580 and Ala583. The BSA desorption has been observed within 1.60 ns. From this, we can list the anchors in order of importance: Lys537, Lys535, Gln542, Thr580 and Ala583 (which was the last to desorb).

We also pull all of nine key residues together, trajectory all_u. It appears that there are five steps in the desorption: (1) The first residues which desorbed are Thr495, Thr580 and Glu494. Next (2), the Glu541 and Ala 583 desorbed from the surface. After that, in step (3) Lys535 is desorbed and Lys537 moved out of the inner layer. Step (4) is observed at $0.57 \mathrm{~ns}$ when Thr539 and Gln542 desorb. The final step (5) is observed when Lys537 lies just above the outer water layer. At $0.88 \mathrm{~ns}$ the BSA molecule lost contact with the silica surface and no more residues anchored the protein to the surface. In the trajectory all_u the list of importance of all residues is slightly different than in trajectory all_noLys537_u. The most significant is Lys537, then Thr539, Gln542, Lys535, Glu541 and Ala583, and finally Thr495, Thr580 and Glu494.

Summarizing the above trajectories, the most important anchors of BSA on $\mathrm{SiO}_{2}$ are: group I which includes only the most important residue, i.e. Lys537; group II with Thr539, Lys535, Glu542; group III with Thr495, Glus541, Glu494 (Ala583 and Thr580 belong either to group II or III). The role of the third group is less important in adsorption process but they probably still moderate the interactions on the surface by interacting with the water layers.

In both trajectories all_noLys537_u and all_u we observe protein desorption from the model silica surface, but the time required for BSA molecule desorption is two times bigger when we pull the key residues without Lys537. During all of the steps described above, no significant conformational changes were observed in the BSA molecule. 


\section{SMD energy analysis}

The energy barriers of the desorption process for trajectory Lys537_u can be assessed by plotting the force acting on the $\mathrm{C} \gamma$-Lys537 atom versus time (Fig. 7A), alongside the displacement of this atom along the direction of the force (Fig. 7B). The first barrier appears at $0.22 \mathrm{~ns}$ when the Lys537 side chain changed orientation and moved out of the inner water layer (F->F'). Then, a distinct subsequent barrier is apparent when Lys537 moves just above the second water layer, which is the move from state F' to $\mathrm{M}$.
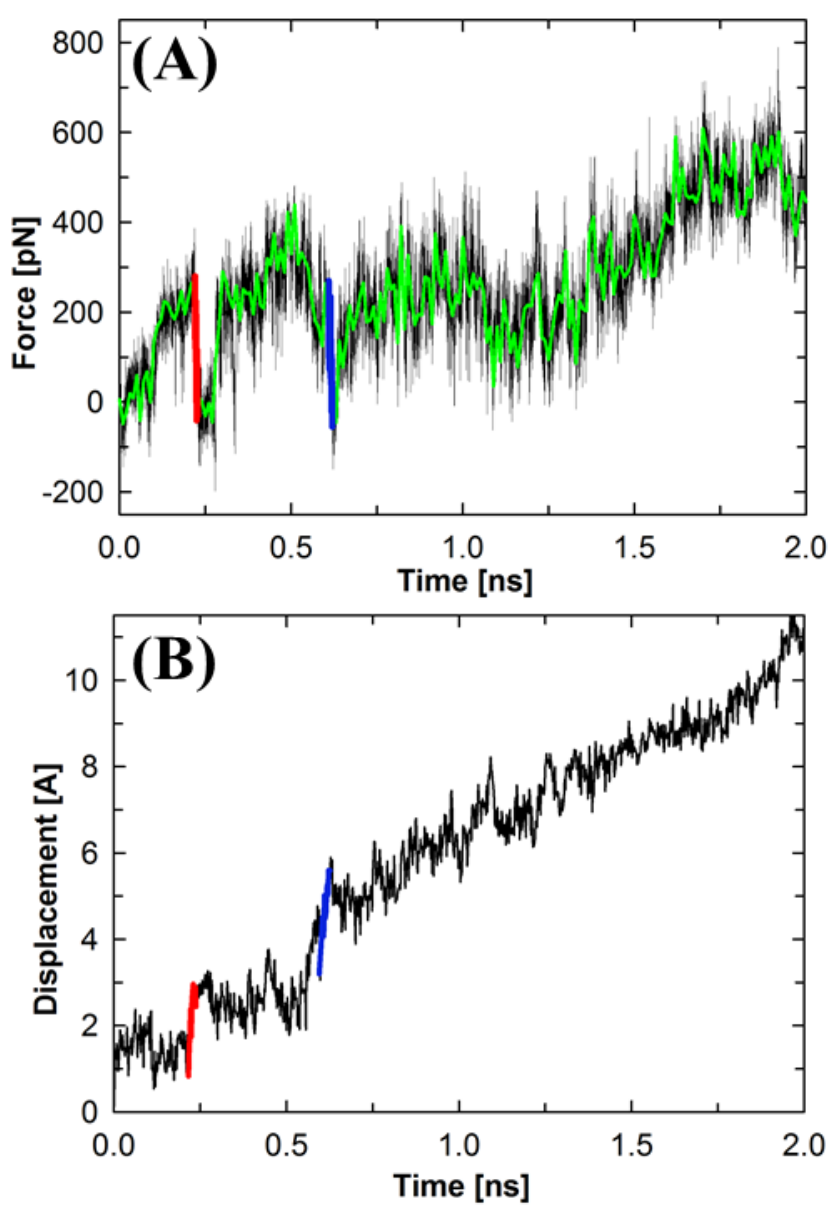
Figure 7. Force (A) and displacement (B) of $\mathrm{C} \gamma$-Lys537 as a function of time (the first 2 ns out of $10 \mathrm{~ns}$ is shown). Transitions between two states F and F' (red line), and between states F' and $\mathrm{M}$ (blue line), are labeled. The green line in (A) shows the running average 0.04 ns window.

The energy barrier $\mathrm{dE}$ for each change in the conformational state of BSA can be calculated from the energy released using the equation:

$$
d E=\left(F_{0}+\frac{d F}{2}\right)\left(\frac{d F}{K}\right)
$$

where $\mathrm{F}_{0}$ is the force at the end of the transition, $\mathrm{dF}$ is the change in force, and $\mathrm{k}$ is the spring constant $(\mathrm{K}=278 \mathrm{pN} / \AA)$. The energy barrier is equal $0.17 \pm 0.1 \mathrm{eV}$ and $0.20 \pm 0.1 \mathrm{eV}$ for the transition from state $\mathrm{F}$ to $\mathrm{F}^{\prime}$, and from state $\mathrm{F}$ ' to $\mathrm{M}$, respectively. We have repeated this analysis for the 3 Lys537_a trajectories and listed the results in Table 2. The average value is $0.16 \pm 0.08$ $\mathrm{eV}$ for transition $\mathrm{F}->\mathrm{F}^{\prime}$ and $0.19 \pm 0.08 \mathrm{eV}$ for $\mathrm{F}^{\prime}->\mathrm{M}$.

We can thus estimate the activation energy barrier for BSA surface diffusion (state $\mathrm{F}$ to $\mathrm{M}$ ) to be $0.35 \pm 0.16 \mathrm{eV}$; as explained above, we believe this will be the same even for the free (unforced) surface diffusion of the adsorbed BSA. From the Arrhenius expression, free surface diffusion should then occur on a timescale of $100 \mathrm{~ns},{ }^{24}$ and indeed this is what we observe in our AD trajectory. ${ }^{5,11}$ From Fig. $7 \mathrm{~A}$, it is apparent that it is difficult to clearly identify other key adsorption events with discrete jumps in the applied force, so we cannot obtain an alternative estimate of the adsorption energy from this data (note that most of the work done by the applied force is dissipated by the solvent). 
Table 2. The energy barriers $\Delta \mathrm{E}$ calculations for the positional changes of BSA by pulling $\mathrm{C} \gamma-$ Lys537 atom in four different directions.

\begin{tabular}{ccc}
\hline Pulling direction & $\Delta$ E stage A [eV] & $\Delta$ E stage B [eV] \\
\hline Lys537_u & $0.17 \pm 0.10$ & $0.20 \pm 0.10$ \\
Lys537_a1 & $0.07 \pm 0.05$ & $0.33 \pm 0.07$ \\
Lys537_a2 & $0.30 \pm 0.09$ & $0.10 \pm 0.07$ \\
Lys537_a3 & $0.11 \pm 0.07$ & $0.15 \pm 0.09$ \\
\hline
\end{tabular}

\section{CONCLUSIONS}

We have studied the interfacial protein dynamics which involve adsorption, slight conformational changes, surface diffusion and desorption. Our work shows that these dynamics are dependent on protein-solvent (notably in the surface water layers) and protein-surface interactions. The binding affinity of BSA to a model hydrophilic silica surface was investigated using MD simulations and supported by SMD; both techniques are key methods for detailed insight into protein adsorption processes on an atomistic level.

Our results support the experimental observation that BSA adsorption is irreversible at physiological $\mathrm{pH} .{ }^{4,11} \mathrm{SMD}$ simulations provided evidence for protein surface diffusion and mobility of adsorbed protein on the silica surface without spontaneous, total desorption. Indeed, our previous MD simulations show that once 
adsorbed, the protein does not desorb on the $500 \mathrm{~ns}$ time scale, but it does freely diffuse across the surface. ${ }^{11}$ The estimated energy of $1.25 \pm 0.4 \mathrm{eV}$ that we obtain for desorption is such that it should not occur even on the experimental time scale of hours, again in agreement with experiments using Quartz Crystal Microbalance and Surface Plasmon Resonance techniques. $^{4,11}$

We have found that protein diffusion on a solid inorganic surface can support its adsorption even when a negatively charged protein adsorbs to a negatively charged surface, and can explain the adsorption process observed experimentally as well as the free surface diffusion of adsorbed BSA we find in MD simulations. ${ }^{11}$ The results obtained for BSA adsorption on the model $\mathrm{SiO}_{2}$ surface can be used as comparative data for complex adsorption and film formation studies using experimental and theoretical techniques for future pharmaceutical applications.

\section{ASSOCIATED CONTENT}

\section{Supporting Information}

The Supporting Information is available free of charge on the ACS Publications website at DOI:

Additional analysis of the energy changes during simulations of the $\mathrm{BSA}^{-\mathrm{SiO}_{2}}$ systems Additional analysis of the BSA structural changes within three trajectories studies: AD, N-AD1 and N-AD2.

\section{AUTHOR INFORMATION}




\section{Corresponding Author}

*E-mail: karina.kubiak@sstrath.ac.uk

Tel. +44 (0)141548 3420

\section{Notes}

The authors declare no competing financial interest.

\section{ACKNOWLEDGEMENTS}

The presented work was supported by Grant NCN OPUS24 2012/07/B/ST5/00767. MD results were obtained using the EPSRC funded ARCHIE-WeSt High Performance Computer (www.archie-west.ac.uk); EPSRC grant no. EP/K000586/1. The European Union Erasmus+ programme (project no. 2015-1-PL01-KA103-014791) is acknowledged for providing financial support for researcher mobility.

\section{REFERENCES}

(1) Raffaini, G.; Ganazzoli, F. Understanding the Performance of Biomaterials through Molecular Modeling: Crossing the Bridge between Their Intrinsic Properties and the Surface Adsorption of Proteins. Macromol. Biosci. 2007, 7, 552-566.

(2) Servoli, E.; Maniglio, D.; Aguilar, M. R.; Motta, A.; San Roman, J.; Belfiore, L. A.; Migliaresi, C. Quantitative Analysis of Protein Adsorption via Atomic Force Microscopy and Surface Plasmon Resonance. Macromol. Biosci. 2008, 8, 1126-1134. 
(3) Pinho, A. C.; Piedade, A. P. Zeta Potential, Contact Angles, and AFM Imaging of Protein Conformation Adsorbed on Hybrid Nanocomposite Surfaces. ACS Appl. Mater. Interfaces 2013, 5, 8187-8194.

(4) Jachimska, B.; Tokarczyk, K.; Łapczyńska, M.; Puciul-Malinowska, A.; Zapotoczny, S. Structure of Bovine Serum Albumin Adsorbed on Silica Investigated by Quartz Crystal Microbalance. Colloids Surfaces A Physicochem. Eng. Asp. 2016, 489, 163-172.

(5) Kubiak-Ossowska, K.; Jachimska, B.; Mulheran, P. A. How Negatively Charged Proteins Adsorb to Negatively Charged Surfaces: A Molecular Dynamics Study of BSA Adsorption on Silica. J. Phys. Chem. B 2016, 120, 10463-10468.

(6) Russell, B. A.; Jachimska, B.; Kralka, I.; Mulheran, P. A.; Chen, Y. Human Serum Albumin Encapsulated Gold Nanoclusters: Effects of Cluster Synthesis on Natural Protein Characteristics. J. Mater. Chem. B 2016, 4, 6876-6882.

(7) Yu, L.; Zhang, L.; Sun, Y. Protein Behavior at Surfaces: Orientation, Conformational Transitions and Transport. J. Chromatogr. A 2015, 1382, 118-134.

(8) Rabe, M.; Verdes, D.; Seeger, S. Understanding Protein Adsorption Phenomena at Solid Surfaces. Adv. Colloid Interface Sci. 2011, 162, 87-106.

(9) Pandey, L. M.; Pattanayek, S. K.; Delabouglise, D. Properties of Adsorbed Bovine Serum Albumin and Fibrinogen on Self-Assembled Monolayers. J. Phys. Chem. C 2013, $117,6151-6160$.

(10) Mathes, J.; Friess, W. Influence of $\mathrm{pH}$ and Ionic Strength on IgG Adsorption to Vials. Eur. J. Pharm. Biopharm. 2011, 78, 239-247.

(11) Kubiak-Ossowska, K.; Tokarczyk, K.; Jachimska, B.; Mulheran, P. A. Bovine Serum 
Albumin Adsorption at a Silica Surface Explored by Simulation and Experiment. J. Phys. Chem. B 2017, 121, 3975-3986.

(12) Mulheran, P. A.; Connell, D. J.; Kubiak-Ossowska, K. Steering Protein Adsorption at Charged Surfaces: Electric Fields and Ionic Screening. RSC Adv. 2016, 6, 73709-73716.

(13) McUmber, A. C.; Randolph, T. W.; Schwartz, D. K. Electrostatic Interactions Influence Protein Adsorption (but Not Desorption) at the Silica-Aqueous Interface. J. Phys. Chem. Lett. 2015, 6, 2583-2587.

(14) Tilton, R. D.; Robertson, C. R.; Gast, A. P. Lateral Diffusion of Bovine Serum Albumin Adsorbed at the Solid-Liquid Interface. J. Colloid Interface Sci. 1990, 137, 192-203.

(15) Langdon, B. B.; Kastantin, M.; Schwartz, D. K. Apparent Activation Energies Associated with Protein Dynamics on Hydrophobic and Hydrophilic Surfaces. Biophys. J. 2012, 102, 2625-2633.

(16) Carlsson, N.; Gustafsson, H.; Thörn, C.; Olsson, L.; Holmberg, K.; Åkerman, B. Enzymes Immobilized in Mesoporous Silica: A Physical-Chemical Perspective. Adv. Colloid Interface Sci. 2014, 205, 339-360.

(17) Pastor, E.; Matveeva, E.; Valle-Gallego, A.; Goycoolea, F. M.; Garcia-Fuentes, M. Protein Delivery Based on Uncoated and Chitosan-Coated Mesoporous Silicon Microparticles. Coll. Surf. B 2011, 88, 601-609.

(18) Mathé, C.; Devineau, S.; Aude, J. C.; Lagniel, G.; Chédin, S.; Legros, V.; Mathon, M. H.; Renault, J. P.; Pin, S.; Boulard, Y.; et al. Structural Determinants for Protein Adsorption/non-Adsorption to Silica Surface. PLoS One 2013, 8, 1-13.

(19) Vilhena, J. G.; Rubio-Pereda, P.; Vellosillo, P.; Serena, P. A.; Pérez, R. Albumin (BSA) 
Adsorption over Graphene in Aqueous Environment: Influence of Orientation, Adsorption Protocol, and Solvent Treatment. Langmuir 2016, 32, 1742-1755.

(20) Pérez-Fuentes, L.; Drummond, C.; Faraudo, J.; Bastos-González, D. Adsorption of Milk Proteins ( $\beta$-Casein and $\beta$-Lactoglobulin) and BSA onto Hydrophobic Surfaces. Materials (Basel). 2017, 10, 1-25.

(21) Wei, T.; Carignano, M. A.; Szleifer, I. Molecular Dynamics Simulation of Lysozyme Adsorption/desorption on Hydrophobic Surfaces. J. Phys. Chem. B 2012, 116, 1018910194.

(22) Mücksch, C.; Urbassek, H. M. Accelerating Steered Molecular Dynamics: Toward Smaller Velocities in Forced Unfolding Simulations. J. Chem. Theory Comput. 2016, 12, $1380-1384$.

(23) Mücksch, C.; Urbassek, H. M. Forced Desorption of Bovine Serum Albumin and Lysozyme from Graphite: Insights from Molecular Dynamics Simulation. J. Phys. Chem. B 2016, 120, 7889-7895.

(24) Kubiak-Ossowska, K.; Mulheran, P. A. Protein Diffusion and Long-Term Adsorption States at Charged Solid Surfaces. Langmuir 2012, 28, 15577-15585.

(25) Wei, T.; Carignano, M. A.; Szleifer, I. Lysozyme Adsorption on Polyethylene Surfaces: Why Are Long Simulations Needed? Langmuir 2011, 27, 12074-12081.

(26) Phillips, J. C.; Braun, R.; Wang, W.; Gumbart, J.; Tajkhorshid, E.; Villa, E.; Chipot, C.; Skeel, R. D.; Kale, L.; Schulten, K. Scalable Molecular Dynamics with NAMD. J. Comput. Chem. 2005, 26, 1781-1802.

(27) Humphrey, W.; Dalke, A.; Schulten, K. MD: Visual MolecularDynamics. J. Mol. 
Graphics 1996,14,33-38. J. Mol. Graph. 1996, 14, 33-38.

(28) Majorek, K. A.; Porebski, P. J.; Dayal, A.; Zimmerman, M. D.; Jablonska, K.; Stewart, A. J.; Chruszcz, M.; Minor, W. Structural and Immunologic Characterization of Bovine, Horse, and Rabbit Serum Albumins. Mol. Immunol. 2012, 52, 174-182.

(29) Freeman. C.L.; Harding, J.H.; Quigley, D.; Rodger, P.M. Simulations of Ovocleidin-17 Binding to Calcite Surfaces and Its Implications for Eggshell Formation. J. Phys. Chem. C 2011, 115, 8175-8183.

(30) Tilton, R.D., Mobility of Biomolecules at Inerfaces. In Biopolymers at Interfaces, Malmsten. M., Marcel Dekker, Inc., New York, 2003, 110, 221-257.

(31) Feder, J. Random Sequential Adsorption. J. Theor. Biol. 1980, 87, 237-254. 